\title{
Comparative evaluation of the QIAsymphony RGQ system with the easyMAG/R-gene combination for the quantitation of cytomegalovirus DNA load in whole blood
}

\author{
Sylvie Pillet, Thomas Bourlet and Bruno Pozzetto*
}

\begin{abstract}
Background: The detection of cytomegalovirus (CMV) DNA in blood is a key feature of the virological surveillance of immunocompromised patients.

Methods: The QIAsymphony RGQ system (QIAGEN S.A.S., France) combines the extraction/distribution steps on QIAsymphony SP/AS instruments with amplification on a Rotor-Gene Q RT-PCR machine. This system was compared to a strategy combining an extraction step on the NUCLISENS easyMAG platform (bioMérieux) with the CMV R-gene kit (Argene) on 100 whole blood specimens collected from immunocompromised patients of the University Hospital of Saint-Etienne, France.

Results: The overall agreement between the two strategies was 86\% (kappa coefficient of 0.67); the 14 discrepant results corresponded to low DNA loads. The 62 samples found positive with both tests were correlated (Pearson $r$ coefficient of $0.70, P<0.01$ ) despite an over quantitation of $0.25 \log _{10}$ copies $/ \mathrm{ml}$ with the easyMAG/Argene strategy $(P<0.001)$. Very close results were also obtained with a commercial panel of 10 samples with CMV loads ranging from 2.36 to $6.41 \log _{10}$ copies $/ \mathrm{ml}$. The inter-run and intra-run variability was consistently lower with the QIAGEN platform.

Conclusions: These results validate the performance of the QIAsymphony RGQ system for the routine quantitation of CMV DNA. This fully-automated platform reduces the hands-on time and improves standardization, traceability and quality control assessment.
\end{abstract}

Keywords: CMV, Viral load, Automation, Molecular biology, Real-time PCR

\section{Background}

Cytomegalovirus (CMV) can cause both early and late multi-organ disease post-transplantantation and remains one of the most important complications after allogeneic transplant. The detection of markers of CMV infection in blood is a key feature of the virological surveillance of immunocompromised patients [1]. Beside antigenemia that is labour intensive, difficult to standardize and requires an immediate analysis of the specimens, molecular methods based on quantitative real-time PCR (RT-PCR) technology are considered to be the main

\footnotetext{
* Correspondence: bruno.pozzetto@univ-st-etienne.fr

Laboratory of Bacteriology-Virology-Hygiene, University Hospital of Saint-Etienne, Saint-Etienne Cedex 02 F-42055, France
}

alternative option for diagnosis of CMV infection, allowing decisions to be made regarding both implementation of pre-emptive therapy, and monitoring response to therapy [2-5]. In nucleic acid amplification techniques, the extraction step is critical and needs to be carefully evaluated notably when different extraction methods are coupled to different PCR techniques [6].

The systematic use of molecular tools for the surveillance of immunocompromised patients needs high throughput machines able to monitor a large number of whole blood or plasma specimens. Automated systems integrating the extraction and amplification steps represent an attractive solution [6-8]. The recently commercialised QIAsymphony RGQ system (QIAGEN S.A.S., 
France) combines the extraction/distribution on the QIAsymphony Sample Preparation (SP) and Assay Setup (AS) modules respectively, together with the amplification step on a Rotor-Gene Q (RGQ) RT-PCR machine using the artus CMV QS-RGQ kit.

In this study, the fully-automated QIAsymphony RGQ system was compared to the molecular strategy currently used in our laboratory for the determination of CMV DNA load from whole blood specimens, which combines extraction with the NUCLISENS easyMAG instrument (bioMérieux, Marcy l'Etoile, France) and amplification using the CMV R-gene kit (Argene, Verniolle, France) as previously validated in our hands [9].

\section{Methods}

\section{Whole blood specimens}

One hundred whole blood specimens were included in the study. Samples were obtained from immunocompromised patients hospitalised at the University Hospital of Saint-Etienne, France, for the following clinical pictures scenarios: bone-marrow graft, kidney transplantation or chronic HIV infection. For all of them, the CMV DNA load was routinely evaluated as part of standard patient care. The study was conducted on the residual clinical specimens stored at $-20^{\circ} \mathrm{C}$. The research was approved by the Ethics Committee of the University Hospital of Saint-Etienne on the $30^{\text {th }}$ of May 2012. For the study, the samples were thawed, divided into 2 aliquots and tested by both systems on the same day. Specimens were then stored at $4^{\circ} \mathrm{C}$ until validated results of quantitation with both amplification methods $(<24 \mathrm{~h})$.

\section{QCMD panel}

The 2010 QCMD (Quality Control for Molecular Diagnostics) program (Glasgow, UK) included 1 negative and 9 positive (AD169 strain) specimens with viral loads ranging from 230 to $2,552,701$ copies/ml, diluted either in CMV-negative human plasma or in virus transport medium. The specimens were reconstituted, separated in aliquot fractions and stored at $-80^{\circ} \mathrm{C}$ as recommended by the QCMD instructions. After thawing, they were tested in the same way as clinical samples with both extraction and amplification systems.

\section{DNA extraction and amplification}

All the experiments were conducted with in vitro diagnosis (IVD) certified kits according to the manufacturers' instructions.

\section{QIAsymphony RGQ system}

Just before extraction, $300 \mu \mathrm{l}$ volumes of the whole blood samples were manually transferred into $2-\mathrm{ml}$ vials. The tubes were placed into 24-tube capacity carrier racks and loaded into the SP module. The extraction step was performed using the QIAsymphony DNA Mini Kit and the Virus Blood 200 protocol with automated eluate dispensing. The internal control of the artus CMV QS-RGQ kit was added into ATE buffer and automatically distributed during the lysis of samples. With this protocol, $200 \mu \mathrm{l}$ volumes of whole blood were extracted and eluted into a total volume of $90 \mu \mathrm{l}$, allowing a minimum accessible volume of $60 \mu$ l. The rack containing the elution tubes was then automatically transferred to the AS module. A $30 \mu \mathrm{l}$ volume of the PCR mix prepared by the AS module using the artus CMV QS-RGQ kit was automatically distributed into RG 72 strip tubes (all reagent and eluate positions are continually cooled) and after distribution of $20 \mu \mathrm{l}$ of template by the AS module, the RG 72 strip tubes were closed and placed manually into the 72-well rotor of the Rotor-Gene Q. The amplification step was performed according to the manufacturer's instructions. Each PCR run included a set of quantitative calibrators corresponding to $10,100,1000$ and 10000 copies/ $\mu$ l of template; the CMV DNA load was calculated from the standard curve and expressed as the number of CMV DNA copies/ml of whole blood (after calculating the input and output volume). The presence of PCR inhibition was detected when the CT value for the internal control of the sample was more than 3 cycles higher than the CT value for the internal control of a negative whole blood control.

\section{Combination of extraction with NUCLISENS easyMAG instrument and amplification with the CMV R-gene kit} Just before extraction, 200- $\mu$ l volumes of the whole blood samples were manually transferred into 4-ml cryotubes containing $2 \mathrm{ml}$ of lysis buffer. The whole extraction process was performed on the NUCLISENS easyMAG instrument according to the previously validated protocol [9]. With this protocol, $200 \mu \mathrm{l}$ of whole blood was extracted and eluted into a total volume of $50 \mu \mathrm{l}$. The eluates were manually transferred into 2 ml-tubes. Fifteen micro liters of ready-to-use PCR mix of the CMV R-gene kit and $10 \mu \mathrm{l}$ of template were manually distributed into a plate stored in a pre-cooled rack. The amplification step was performed using an ABI7500 instrument (Applied Biosystems) according to Argene's instructions. Each PCR run included a set of quantitative calibrators corresponding to 5, 50, 500 and 5000 copies/ $\mu \mathrm{l}$ of template; the CMV DNA load was calculated from the standard curve and expressed as the number of CMV DNA copies/ml of whole blood. The presence of PCR inhibition was detected when the CT value for the internal control of the sample was more than 3 cycles higher than the CT value for the internal control of a negative whole blood control.

\section{Analytic performances of the QIAGEN workflow}

The analytical performances of the QIAGEN workflow were studied with frozen pooled blood samples from 
patients of our hospital with known CMV DNA load values. The limit of detection of the test was assessed using seven $0.5-\log _{10}$ dilution series of CMV DNA positive whole blood in CMV DNA negative blood to yield concentrations ranging from 14 to 10000 CMV DNA copies $/ \mathrm{ml}$. Each measure was tested on 5 replicate aliquots.

The reproducibility of the QIAGEN system was determined in comparison with the easyMAG/Argene technique using 4 pools prepared from positive specimens and exhibiting different DNA loads: one low $\left(<3 \log _{10}\right.$ copies $/ \mathrm{ml}$ ), two intermediate (between 3 and $4.5 \log _{10}$ copies $/ \mathrm{ml}$ ) and one high (> $4.5 \log _{10}$ copies $\left./ \mathrm{ml}\right)$. For each concentration, 8 frozen aliquots were tested, 4 for intra-run reproducibility assay and 4 for inter-run reproducibility assay (performed each on a different day).

\section{Correlation}

The correlation analysis between the two systems was performed using a set of paired aliquots of whole blood specimens. The extraction and amplification steps were performed the same day for the same sample with both techniques.

\section{Statistical analysis}

The lower limit of detection of the QIAGEN assay was determined by probit analysis [10]. The agreement between methods' results was tested by the kappa coefficient. Bivariate correlation analysis was performed with the Pearson $r$ coefficient and a two-tailed test of significance. Differences in mean values were analysed by the paired $\mathrm{t}$ test. $P$ values of 0.05 were considered as the threshold of significance.

\section{Results}

Limit of detection and reproducibility of the QIAGEN workflow

The limit of detection of the QIAGEN workflow, calculated by probit analysis, was 72 copies $/ \mathrm{ml}$, with a confidence of $95 \%$ (Figure 1). By comparison to the easyMAG/ Argene system, the inter-run and intra-run variability was consistently lower with the QIAGEN platform, notably for the low and intermediate viral loads (Table 1).

\section{QCMD panel analysis}

Ten samples belonging to the 2010 QCMD panel were tested in duplicate with the two systems. The negative sample was correctly assigned by both techniques. The results for the 9 positive samples are illustrated in Figure 2: the two systems exhibited the same range of quantitation, despite the fact that the viral load was consistently higher with the easyMAG/Argene method.

\section{Correlation between the test systems in clinical specimens}

From the 100 clinical samples tested comparatively with both systems, 3 needed to be tested again for an invalid internal control value, 1 for easyMAG/Argene and 2 for QIAGEN and could be validated after retest.

Of the 100 pairs of clinical samples, 62 were positive with both methods and 24 were negative, which corresponds to a substantial agreement of $86 \%$ (kappa coefficient of 0.67). Fourteen samples exhibited discrepant results: 11 were tested positive with the QIAGEN platform and negative with the easyMAG/Argene method and 3 samples exhibited the opposite result; all of these discrepant results corresponded to viral loads $<2.5 \log$ copies $/ \mathrm{ml}$.

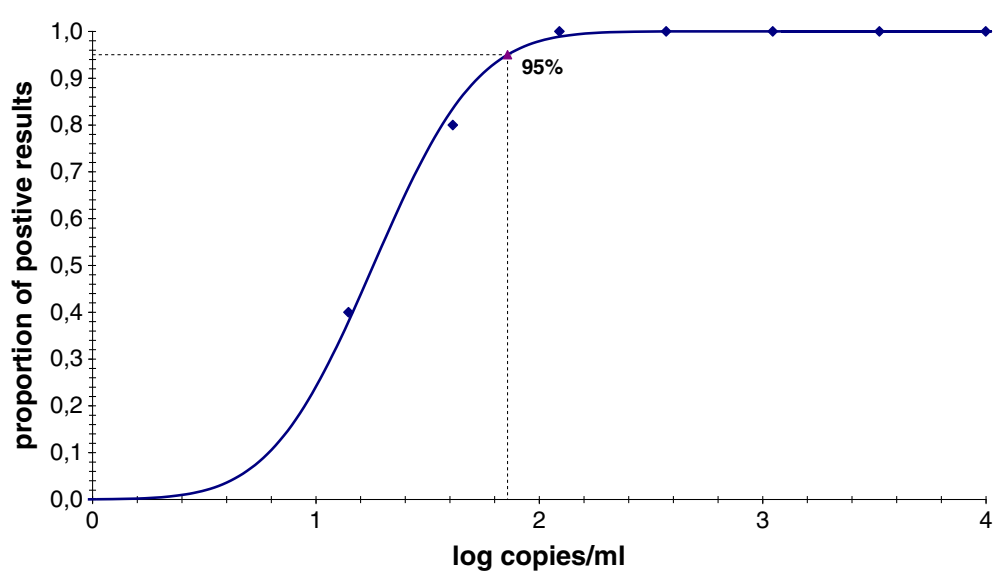

Figure 1 Probit curve used to calculate the limit of detection of the QIAsymphony RGQ method. Seven 0.5- $\log _{10}$ dilution series of CMV DNA positive whole blood in CMV DNA negative blood (lozenges) to yield concentrations ranging from 14 to 10000 CMV DNA copies/ml were tested 5 times each. The limit of detection at $95 \%$ was extrapolated from the sigmoid curve. 
Table 1 Intra- and inter-run reproducibility of the two strategies compared in the study on 4 replicates of each pool

\begin{tabular}{llllll}
\hline & \multicolumn{4}{c}{ Mean (\% coefficient of variation)* } \\
\cline { 2 - 3 } & \multicolumn{2}{c}{ QIAGEN system } & & easyMAG/Argene combination \\
\cline { 2 - 3 } \cline { 5 - 6 } \cline { 5 - 6 } Intra-run & Inter-run & & Intra-run & Inter-run \\
\hline Pool 1 & $2.30(6.39)$ & $2.47(20.54)$ & & $2.82(10.48)$ & $2.48(23.75)$ \\
Pool 2 & $3.51(2.13)$ & $3.53(2.21)$ & & $4.54(9.75)$ & $4.00(15.71)$ \\
Pool 3 & $3.70(2.61)$ & $3.58(2.84)$ & & $4.34(4.12)$ & $4.33(6.17)$ \\
Pool 4 & $4.83(2.83)$ & $4.72(3.02)$ & & $5.12(1.18)$ & $5.12(0.89)$ \\
\hline
\end{tabular}

${ }^{*} \log _{10}$ copies $/ \mathrm{ml}$ of whole blood.

The 62 samples found positive with both tests were used to draw the correlation curve (Figure $3 \mathrm{~A}$ ) and the Bland-Altman plot (Figure 3B). The Pearson $r$ coefficient was $0.70(P<0.01)$ and the mean difference in viral loads was $0.25 \log _{10}$ copies $/ \mathrm{ml}$ to the benefit of the easyMAG/Argene strategy $(P<0.001)$. Samples corresponding to low CMV DNA loads exhibited a high variability. Among those with a CMV DNA load above 1000 copies/ml, 6 exhibited discrepancy of at least 0.5 log copies/ ml: 5 with CMV load higher by the easyMAG/Argene method, one of them being below the mean of the difference - 2 SD in Figure 3B, and one with CMV load higher by the QIAGEN platform.

The time necessary to complete a run was evaluated to 3.5 and 3.7 hours for the easyMAG/Argene strategy and the QIAGEN system, respectively. However, the hands-on time was approximately of 0.3 hour for the QIAGEN system versus 1 hour for the easyMAG/Argene strategy.

\section{Discussion}

The automation of molecular techniques performed on a large scale is an important challenge in clinical virology. The QIAGEN workstation consisting of the QIAsymphony SP and AS modules that has been recently commercialised allows the successive achievement of nucleic acid purification from various samples (SP module) and the distribution of the extract combined with master mix in PCR tubes (AS module). This automated system has been recently evaluated for the detection of enteric pathogens in faecal samples $[11,12]$ and the quantitation of hepatitis $C$ viral load [13]. In addition, three other studies evaluated the QIAsymphony SP extraction module alone for Epstein-Barr virus [14], HIV viral load [15] and a panel of different viruses [16].

In this study, we evaluated the QIAGEN workstation combined with the RGQ platform for the quantitation of CMV DNA with the CMV artus CMV QS-RGQ kit from $200 \mu \mathrm{l}$ of whole blood. Different versions of this amplification kit have been shown to yield comparable results of CMV DNA load with other molecular techniques either in EDTA-plasma $[17,18]$ or in whole blood [19].

Regarding the extraction step, previous studies evaluated the QIAsymphony system for the quantitation of CMV DNA in blood under different configurations: Raggam et al. [20] tested the QIAsymphony SP module in combination with the R-gene kit from $200 \mu \mathrm{l}$ of whole blood;

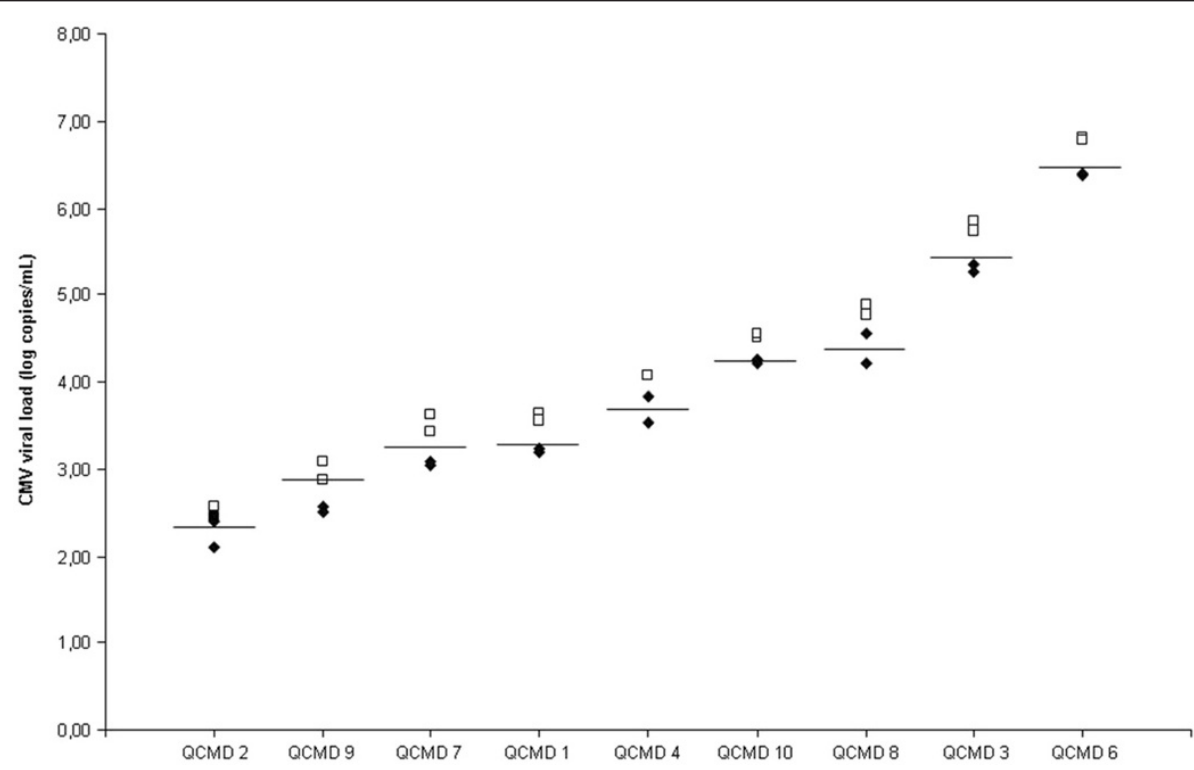

Figure 2 CMV DNA load obtained with both methods for the samples of the 2010 QCMD program. Viral loads obtained with the QIAsymphony RGQ system and the easyMAG/Argene combination are represented with diamonds and squares, respectively. The measures were performed in duplicate. The horizontal bars show the theoretical viral loads given by the manufacturer of the proficiency panel. 

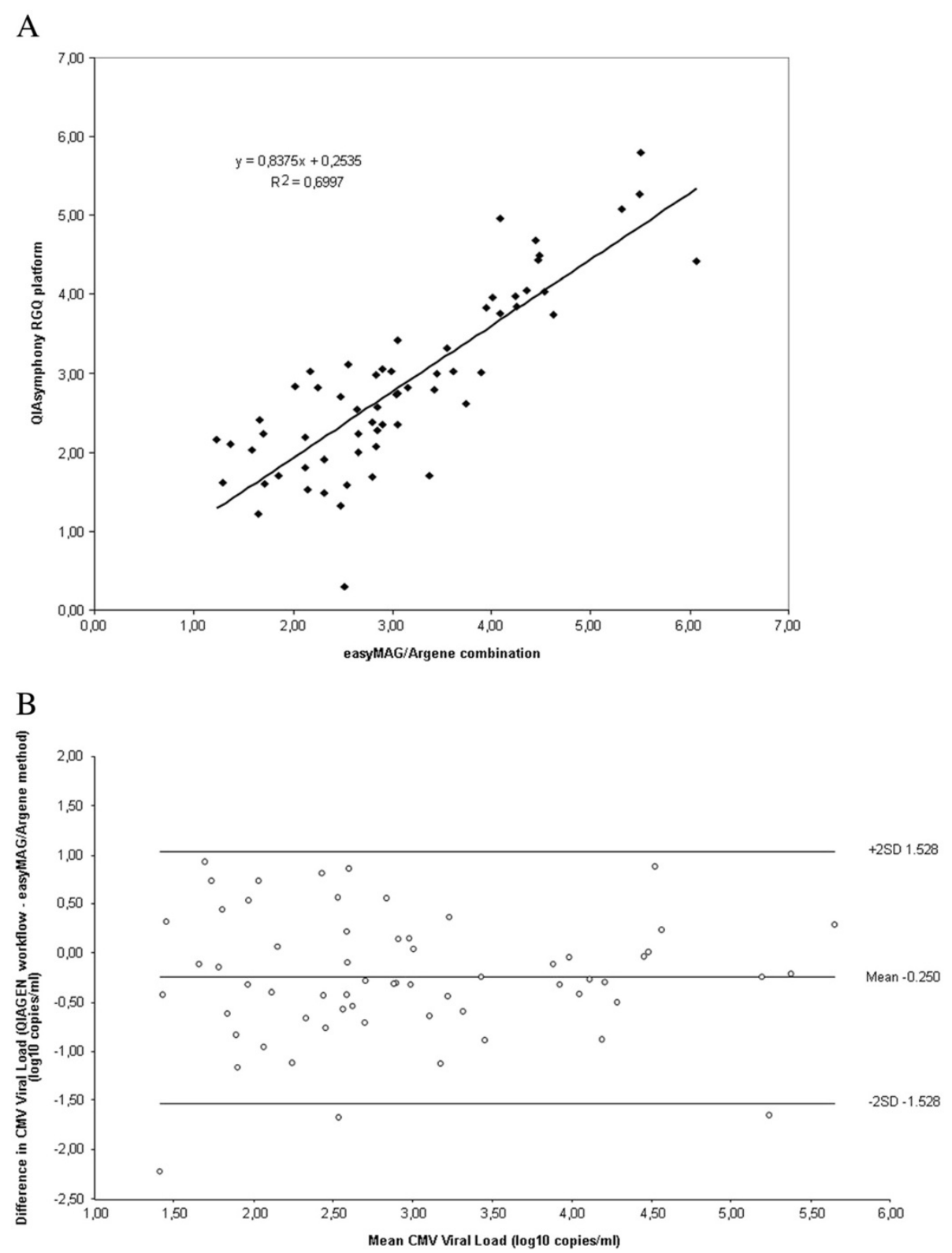

Figure 3 Correlation analysis using clinical samples. (A) Comparison of CMV DNA load by QIAsymphony RGQ platform and easyMAG/ Argene combination using whole blood samples ( $\mathrm{N}=62$ ). The results are expressed in $\log _{10}$ copies $/ \mathrm{ml}$. (B) Difference in quantitation between QIAsymphony RGQ platform and easyMAG/Argene combination. Each point represents the difference observed between the results of the 2 methods against their mean. The standard deviation (SD) is 0.639 .

Miller et al. [21] evaluated the same extraction module with the Roche kit from $1 \mathrm{ml}$ of blood serum; finally, Forman et al. [22] tested the same configuration as the one used in the present study but from a different matrix (1.2 $\mathrm{ml}$ of blood plasma). The limit of detection of these different methodologies was 148, 90, and 23 CMV DNA copies/ml, respectively; the value of 72 copies/ml obtained in the current study with the QIAsymphony RGQ system using a small volume $(200 \mu \mathrm{l})$ of whole blood was very close to that of the three former studies and to that specified in the QIAGEN handbook of the kit (164.6 copies $/ \mathrm{ml}$ ). It was much lower than that mentioned for the R-gene technique in the manufacturer's handbook but that had been obtained with a MagNA Pure Compact automate $(555$ copies $/ \mathrm{ml})$.

By reference to the strategy routinely used in our laboratory that we had previously evaluated favourably [9], the QIAsymphony RGQ system was well correlated, despite a slight translation of CMV DNA loads to the benefit of the easyMAG/R-gene couple. By contrast, a better 
sensitivity was obtained with the QIAsymphony RGQ system for low positive samples as illustrated by the distribution of positive discrepant samples (11 for the QIAGEN platform and only 3 for the easyMAG/Argene method). Furthermore, the inter-assay and intra-assay variability was shown to be lower with the QIAsymphony RGQ system because of its complete automation, whereas the easyMAG/R-gene combination includes several manual steps (sample preparation, addition of magnetic silica, transfer of eluates into microtubes, preparation and distribution of PCR mix, calibrators and samples...).

\section{Conclusion}

In addition to fulfilling excellent technical performances (linearity comprised between $1 \times 10^{3}$ and $5 \times 10^{7}$ copies $/ \mathrm{ml}$, and $95 \%$ limit of detection of 164.6 copies $/ \mathrm{ml}$ for whole blood according to the manufacturer's specifications), the QIAsymphony RGQ system offers a fully-automated workflow with reduction of hands-on time and improvement in standardisation, traceability and quality control assessment. It appears particularly adapted to the routine surveillance of CMV DNA load in immunocompromised patients.

\section{Competing interest}

Except the fact that the reagents were provided free of charge by QIAGEN

S.A.S., France, the authors declare no conflict of interest neither with this society nor with any other.

\section{Authors' contribution}

SP and BP designed the study; SP made the experiments; TB contributed to the statistical analysis and to the critical reading of the manuscript; SP and BP wrote the paper. All authors read and approved the final manuscript.

\section{Acknowledgements}

We are grateful to Monique Feneyrolle, Jannick Granger, Nathalie Plotton and Sandra Veyre for excellent technical assistance. We thank Gavin Wall from QIAGEN GmbH, Germany, for helpful scientific discussions. Reagents required for this study were supported by QIAGEN S.A.S., France.

Received: 30 December 2011 Accepted: 3 October 2012

Published: 9 October 2012

\section{References}

1. Atkinson C, Emery VC: Cytomegalovirus quantitation: Where to next in optimising patient management? J Clin Virol 2011, 51:223-228.

2. Boeckh M, Ljungman P: How we treat cytomegalovirus in hematopoietic cell transplant recipients. Blood 2009, 113:5711-5719.

3. Halfon P, Berger P, Khiri H, Martineau A, Pénaranda G, Merlin M, Faucher C: Algorithm based on CMV kinetics DNA viral load for preemptive therapy initiation after hematopoietic cell transplantation. J Med Virol 2011, 83:490-495.

4. Kotton CN, Kumar D, Caliendo AM, Asberg A, Chou S, Snydman DR, Allen U, Humar A: International consensus guidelines on the management of cytomegalovirus in solid organ transplantation. Transplantation 2010, 89:779-795.

5. Ljungman P, de la Camara R, Cordonnier C, Einsele H, Engelhard D, Reusser P, Styczynski J, Ward K: Management of CMV, HHV-6, HHV-7 and Kaposisarcoma herpesvirus (HHV-8) infections in patients with hematological malignancies and after SCT. Bone Marrow Transplant 2008, 42:227-240.

6. Bravo D, Clari MÁ, Costa E, Muñoz-Cobo B, Solano C, José Remigia M, Navarro D: Comparative Evaluation of Three Automated Systems for DNA Extraction in Conjunction with Three Commercially Available Real-Time PCR Assays for Quantitation of Plasma Cytomegalovirus DNAemia in Allogeneic Stem Cell Transplant Recipients. J Clin Microbiol 2011, 49:2899-2904.
7. Kerschner H, Bauer C, Schlag P, Lee S, Goedel S, Popow-Kraupp T: Clinical evaluation of a fully automated CMV PCR assay. J Clin Virol 2011, 50:281-286.

8. Mengelle C, Mansuy J-M, Da Silva I, Davrinche C, Izopet J: Comparison of 2 highly automated nucleic acid extraction systems for quantitation of human cytomegalovirus in whole blood. Diagn Microbiol Infect Dis 2011, 69:161-166

9. Pillet $\mathrm{S}$, Bourlet $\mathrm{T}$, Pozzetto B: Comparative evaluation of a commercially available automated system for extraction of viral DNA from whole blood: application to monitoring of epstein-barr virus and cytomegalovirus load. J Clin Microbiol 2009, 47:3753-3755.

10. Sakuma M: Probit analysis of preference data. Appl Entomol Zool 2010, 33:339-47.

11. van Zanten E, Wisselink GJ, de Boer W, Stoll S, Alvarez R, Kooistra-Smid AMD: Comparison of the QIAsymphony automated nucleic acid extraction and PCR setup platforms with NucliSens easyMAG and Corbett CAS-1200 liquid handling station for the detection of enteric pathogens in fecal samples. J Microbiol Methods 2011, 84:335-340.

12. van Zanten E, Wisselink GJ, Stoll S, Alvarez R, Kooistra-Smid AMD: Evaluation of a shortened QIAsymphony DNA extraction protocol for stool samples using a multiplex real-time PCR for the detection of enteric pathogens. J Microbiol Methods 2011, 84:365-367.

13. Paba P, Fabeni L, Perno CF, Ciotti M: Performance evaluation of the Artus hepatitis C virus QS-RGQ assay. J Virol Methods 2012, 179:77-80.

14. Laus S, Kingsley LA, Green M, Wadowsky RM: Comparison of QIAsymphony automated and QIAamp manual DNA extraction systems for measuring epstein-barr virus DNA load in whole blood using real-time PCR. J Mol Diagn 2011, 13:695-700.

15. Sandres-Sauné K, Raymond S, Nicot F, Boineau J, Pasquier C, Izopet J: Evaluation of the QIAsymphony ${ }^{\mathrm{TM}}$ SP and Artus ${ }^{\mathrm{TM}}$ RealTime extractionquantitation systems for measuring HIV-1 virus load. J Clin Virol 2011, 52:349-352.

16. Lee AV, Atkinson C, Manuel RJ, Clark DA: Comparative evaluation of the QIAGEN QIAsymphony ${ }^{\circledR}$ SP system and bioMérieux NucliSens easyMAG automated extraction platforms in a clinical virology laboratory. J Clin Virol 2011, 52:339-343.

17. Caliendo AM, Ingersoll J, Fox-Canale AM, Pargman S, Bythwood T, Hayden MK, Bremer JW, Lurain NS: Evaluation of real-time PCR laboratorydeveloped tests using analyte-specific reagents for cytomegalovirus quantitation. J Clin Microbiol 2007, 45:1723-1727.

18. Hanson KE, Reller LB, Kurtzberg J, Horwitz M, Long G, Alexander BD: Comparison of the Digene Hybrid Capture System Cytomegalovirus (CMV) DNA (version 2.0), Roche CMV UL54 analyte-specific reagent, and QIAGEN RealArt CMV LightCycler PCR reagent tests using AcroMetrix OptiQuant CMV DNA quantitation panels and specimens from allogeneic-stem-cell transplant recipients. J Clin Microbiol 2007, 45:1972-1973.

19. Michelin BDA, Hadzisejdic I, Bozic M, Grahovac M, Hess M, Grahovac B, Marth $\mathrm{E}$, Kessler HH: Detection of cytomegalovirus (CMV) DNA in EDTA whole-blood samples: evaluation of the quantitative artus CMV LightCycler PCR kit in conjunction with automated sample preparation. J Clin Microbiol 2008, 46:1241-1245.

20. Raggam RB, Bozic M, Salzer HJF, Hammerschmidt S, Homberg C, Ruzicka K, Kessler HH: Rapid quantitation of cytomegalovirus DNA in whole blood by a new molecular assay based on automated sample preparation and real-time PCR. Med Microbiol Immunol 2010, 199:311-316.

21. Miller S, Seet H, Khan Y, Wright C, Nadarajah R: Comparison of QIAGEN automated nucleic acid extraction methods for CMV quantitative PCR testing. Am J Clin Pathol 2010, 133:558-563.

22. Forman M, Wilson A, Valsamakis A: Cytomegalovirus DNA quantitation using an automated platform for nucleic Acid extraction and real-time PCR assay setup. J Clin Microbiol 2011, 49:2703-2705.

\section{doi:10.1186/1743-422X-9-231}

Cite this article as: Pillet et al:: Comparative evaluation of the

QIAsymphony RGQ system with the easyMAG/R-gene combination for the quantitation of cytomegalovirus DNA load in whole blood. Virology Journal 2012 9:231. 